BMJ Open

Diabetes

Research

\& Care

\title{
Diabetic hand: prevalence and incidence of diabetic hand problems using data from 1.1 million inhabitants in southern Sweden
}

\author{
Mattias Rydberg (10 , ${ }^{1,2}$ Malin Zimmerman, ${ }^{1,2}$ Anders Gottsäter, ${ }^{3}$ \\ Ann-Marie Svensson, ${ }^{4,5}$ Katarina Eeg-Olofsson (D) , , $^{5,6}$ ars B Dahlin ${ }^{1,2}$
}

To cite: Rydberg M, Zimmerman M, Gottsäter A, et al. Diabetic hand: prevalence and incidence of diabetic hand problems using data from 1.1 million inhabitants in southern Sweden. BMJ Open Diab Res Care 2022;10:e002614. doi:10.1136/

bmjdrc-2021-002614

- Additional supplemental material is published online only. To view, please visit the journal online (http://dx.doi. org/10.1136/bmjdrc-2021002614).

Received 28 September 2021 Accepted 23 December 2021

Check for updates

C Author(s) (or their employer(s)) 2022. Re-use permitted under CC BY-NC. No commercial re-use. See rights and permissions. Published by BMJ.

For numbered affiliations see end of article.

Correspondence to Dr Mattias Rydberg; mattias.rydberg@med.lu.se

\section{ABSTRACT}

Introduction 'The diabetic hand' has traditionally referred to hand complications due to diabetes mellitus (DM), including trigger finger (TF) and Dupuytren's disease (DD). Recent publications have also proposed DM as a risk factor for carpal tunnel syndrome (CTS), ulnar nerve entrapment (UNE), and possibly osteoarthritis (OA) of the first carpometacarpal (CMC-1) joint. This study aimed to explore prevalence and incidence of diabetic hand complications among the population in southern Sweden. Research design and methods Approximately 1.1 million inhabitants in the region of Skåne aged $\geq 18$ years, whereof 50000 with DM, were included. Data on incident CTS, UNE, TF, DD, and OA of the CMC-1 joint between 2004 and 2019 were collected from the Skåne Healthcare Register and cross-linked with the National Diabetes Register. Prevalences on December 31, 2019 and 10-year incidence ratios were calculated for type 1 diabetes (T1D), type 2 diabetes (T2D), and the population without DM, stratified for sex. Prevalence ratios and incidence rate ratios with $95 \%$ Cls were used for group comparisons.

Results The prevalences of all five studied diagnoses were higher in both men and women with T1D and T2D $(p<0.01)$ and both T1D and T2D had more concomitant prevalent diagnoses $(p<0.0001)$. The 10 -year incidence rates of all diagnoses were higher among $\mathrm{T} 1 \mathrm{D}$ and $\mathrm{T} 2 \mathrm{D}$ $(p<0.0001)$, except $0 A$ of the CMC- 1 joint in men with T1D $(\mathrm{p}=0.055)$.

Conclusions CTS, UNE, and possibly also OA of the CMC-1 joint should be included together with TF and DD when referring to 'the diabetic hand'. The incidence of hand disorders was up to eight times higher among T1D, and both T1D and T2D had more concomitant prevalent diagnoses compared with the population without DM. Future studies should elucidate the pathophysiology behind diabetic hand complications to enable development of effective preventive measures in patients with diabetes.

\section{INTRODUCTION}

The concept of hand complications due to diabetes mellitus (DM) was first introduced during the 1970s, although the term 'the diabetic hand' did not occur in the literature until much later. ${ }^{1}$ Initially, 'the diabetic hand' included diagnoses, such as trigger finger

\section{Significance of this study}

What is already known about this subject?

- Diabetes has been proposed as a risk factor for common hand disorders, for example, trigger finger and Dupuytren's disease.

- Less is known regarding the impact of diabetes on osteoarthritis of the hand and compression neuropathies, for example, carpal tunnel syndrome and ulnar nerve entrapment.

What are the new findings?

- All the studied diagnoses in this study were more prevalent among individuals with type 1 diabetes and type 2 diabetes, compared with the population without diabetes.

- The incidence of hand disorders was up to eight times higher among the population with type 1 diabetes compared with the population without diabetes.

- Both population with type 1 and type 2 diabetes had more concomitant prevalent diagnoses when compared with a population without diabetes mellitus.

How might these results change the focus of research or clinical practice?

- The results from this study indicate that complications to diabetes mellitus are common in the hand and many individuals suffer from more than one diagnose.

- Focus should now be on preventative measures against the diabetes epidemic in order to prevent complications such as the diabetic hand.

(TF), limited joint mobility, and Dupuytren's disease (DD) ${ }^{12}$ However, recent publications have suggested that DM is also a risk factor for upper extremity compression neuropathies (CNs), that is, carpal tunnel syndrome (CTS) and ulnar nerve entrapment (UNE), ${ }^{34}$ and possibly also osteoarthritis (OA) of the first carpometacarpal (CMC-1) joint. ${ }^{5}$

DM is a growing global health issue, with a prevalence of 420 million people or $9 \%$ 
globally in $2014,{ }^{6}$ and 23 million incident cases every year. ${ }^{7}$ Exactly how much higher the prevalences and incidences of the aforementioned diagnoses are among the population with DM is not known, although the number of hand diagnoses is likely to rise in the coming decades due to the increasing prevalence of DM. In order to provide relevant patient information and preventative measures, it is important to clarify how DM complications manifest not only in relation to cardiovascular, renal, or eye complications, but also regarding hand complications, in view of individual suffering as well as costs for society.

Thus, the aim of this study was to further explore prevalences and incidences of CTS, UNE, TF, DD, and OA of the CMC-1 joint among the 1.1 million inhabitants in the region of Skåne in southern Sweden, in persons with or without diabetes, in order to better describe and define the diagnoses associated with 'the diabetic hand'.

\section{RESEARCH DESIGN AND METHODS}

\section{Study population}

The population studied was all approximately 1.1 million residents living in the region of Skåne in southern Sweden during 2004-2019, aged $\geq 18$ years and all registered in the National Diabetes Register (NDR). ${ }^{8}$ The region of Skåne provides healthcare on both primary and specialized secondary care, and there are both public and private caregivers. All caregivers are obliged to register patient diagnoses in the Skåne Healthcare Register (SHR) ${ }^{9}$ Furthermore, there are approximately 50000 inhabitants in the region with DM, all registered in the NDR. ${ }^{8}$

\section{Data sources}

The SHR collects data from all caregivers in the region of Skåne using the unique 10-digit personal identification number assigned to all inhabitants in Sweden. Since 1997, all diagnoses in the region are classified according to International Classification of Disease (ICD) 10; however, the SHR also registers surgical and procedure codes. Furthermore, the SHR registers the type of caregiver (primary care, specialized hospital-based care), age at diagnosis and sex. The register covers the vast majority of all physician visits with an assigned diagnose code and there have been several performed validation studies. ${ }^{9}$

The NDR was founded in 1996 in order to improve treatment and quality of life among inhabitants living with DM in Sweden. In short, the NDR data from all primary and secondary diabetes care in Sweden cover over $90 \%$ of all persons with DM in Sweden. ${ }^{10}$ The NDR collects data on clinical characteristics, treatments and risk factors, for example, diabetes duration, type of diabetes, medications, glycemic control, blood lipid levels and body mass index (BMI). In this study, only two types of diabetes, that is, type 1 diabetes (T1D) and type 2 diabetes (T2D), were used. The NDR, data collection and definitions of T1D and T2D have previously been described in detail. ${ }^{1011}$
Finally, for yearly population data, the Swedish Population Register (https://www.scb.se/en/) was used. All three registers were linked using the inhabitant's unique personal identification number.

\section{Case and group definition}

All inhabitants in the region of Skåne aged $\geq 18$ years, diagnosed with either CTS, UNE, TF, DD, or OA of the CMC-1 joint between 2004 and 2019, were registered in SHR and included in the study. The following ICD-10 codes for respective diagnose were used: CTS: G56.0; UNE: G56.2 G562C, G562D, G562X; TF: M65.3; DD: M72.0; and OA of the CMC-1 joint: M18.0, M18.1, M18.9. Data from both primary and specialized secondary care were used; however, no surgical or procedure codes were used in this study.

\section{Statistical analysis}

All calculations were made separately for each of the aforementioned diagnoses and separately for individuals with T1D, T2D, and without DM, thus enabling group comparisons. For prevalence calculation, we only included the inhabitants still alive and living in Skåne on December 31, 2019 who had been diagnosed during 2004-2019. Sex-stratified point prevalence estimates were calculated and analysed; and for group comparison, prevalence ratios (PRs) with 95\% CIs, using the Taylor series method, were calculated. ${ }^{12}$ For calculation of concomitant prevalent diagnoses (maximum five diagnoses), all individuals with at least one diagnosis were grouped in a dichotomous variable of either one or two or more diagnoses. For comparisons between T1D, T2D, and non-DM groups, the $\mathrm{X}^{2}$ test was used.

For incidence calculation, we included inhabitants with first time diagnoses of the respective conditions during the period from 2010 to 2019, without a history of that specific diagnosis during 2004-2009. Age-stratified and sex-stratified incidence ratios (IRs) were calculated and for group comparisons, sex-stratified incidence rate ratios (IRRs) with 95\% CI, using the Byar's approximation method, were calculated and analyzed. ${ }^{13}$ When calculating the population at risk, the total number of inhabitants in the region each year from 2010 to 2019 was used, subtracting the number of inhabitants with DM. For population at risk calculation among the population with DM, the total number of individuals with T1D and T2D each year during 2010-2019 was used. The age of each inhabitant as per December 31 of respective year was used when calculating the age-stratified incidence. For incidence calculation, the DM diagnosis had to be established prior to or during the same year as the hand diagnosis. For all calculations, a two-sided $p$ value of $<0.05$ was considered significant.

All statistical calculations were made using IBM SPSS statistics for MAC V.27 and Open Epi (www.openepi. com). ${ }^{14}$ All figures were made using Microsoft Excel for MAC V.11.5 (Microsoft Corporations, Redmond, Washington, USA). 
Table 1 Population demographics as of December 31, 2019 of the inhabitants in region Skåne aged $\geq 18$ years, stratified for diabetes status

\begin{tabular}{|c|c|c|c|}
\hline $\begin{array}{l}\text { Population group } \\
\text { (age intervals, years) }\end{array}$ & Without diabetes & Type 1 diabetes & Type 2 diabetes \\
\hline Total (18+) & 1041336 & 6812 & 49395 \\
\hline $\begin{array}{l}\text { Women }(\%)^{*} \\
\text { Total }(18+)\end{array}$ & $528310(50.7)$ & 3045 (44.7) & 20453 (41.4) \\
\hline $18-29$ & 89645 & 577 & 80 \\
\hline 30-39 & 95163 & 426 & 304 \\
\hline $40-49$ & 85619 & 486 & 1020 \\
\hline $50-59$ & 84324 & 527 & 2740 \\
\hline $60-69$ & 67723 & 483 & 4938 \\
\hline $70-79$ & 61534 & 409 & 6955 \\
\hline $80+$ & 44302 & 137 & 4416 \\
\hline $\begin{array}{l}\text { Men }(\%)^{*} \\
\text { Total }(18+)\end{array}$ & 513026 (49.3) & 3767 (55.3) & $28942(58.6)$ \\
\hline 18-29 & 95829 & 684 & 64 \\
\hline $30-39$ & 95785 & 550 & 351 \\
\hline $40-49$ & 87690 & 653 & 1470 \\
\hline $50-59$ & 84447 & 682 & 4312 \\
\hline $60-69$ & 63790 & 586 & 7827 \\
\hline $70-79$ & 53947 & 508 & 10290 \\
\hline $80+$ & 31538 & 104 & 4628 \\
\hline
\end{tabular}

*Percentage of sex distribution.

\section{RESULTS}

Age-stratified and sex-stratified population demographics as per December 31, 2019 among the inhabitants in Skåne with T1D, T2D, and without DM, respectively, are presented in table 1.

There were in total 551808 women aged $\geq 18$ years, whereof 3045 with T1D and 20453 with T2D, and in total 545735 men aged $\geq 18$ years, whereof 3767 with T1D and 28942 with T2D.

\section{Prevalence 2004-2019}

The prevalence data for CTS, TF, UNE, DD, and OA of the CMC-1 joint, respectively, are presented in table 2, stratified for sex and diabetes status.

The prevalences of all studied diagnoses were higher among the population with T1D and T2D when compared with the population without DM, with PR spanning from 1.8 (95\% CI: 1.4 to $2.4, \mathrm{p}<0.01$ ) for $\mathrm{OA}$ of the $\mathrm{CMC}-1$ joint to 9.4 (95\% CI: 8.6 to 10.3 , $\mathrm{p}<0.0001)$ for TF among women with T1D. Figure 1 presents prevalence data for the respective diagnoses with $95 \% \mathrm{CI}$, stratified for sex and diabetes status.

The proportions of the population with a second, concomitant prevalent diagnosis were higher among both individuals with T1D (women 34.0\%; 95\% CI: 30.6\% to $37.5 \%$, men $24.1 \%$; $95 \%$ CI: $20.7 \%$ to $27.8 \%$ ) and T2D (women $22.4 \%$; $95 \%$ CI: $21.1 \%$ to $23.8 \%$, men $18.8 \%$; $95 \%$ CI: $17.5 \%$ to $20.1 \%$ ), compared with the population without DM (women 16.7\%; 95\% CI: $16.3 \%$ to $17.1 \%$, men $13.5 \%$; $95 \%$ CI: $13.0 \%$ to $14.1 \%$ ) ( $X^{2} p<0.0001$ for all analyses).

\section{IRs 2010-2019}

The IRs per 10000 person-years for CTS, UNE, TF, DD, and $\mathrm{OA}$ of the CMC-1 joint, respectively, are presented in table 3 , stratified by sex and diabetes status with IRR with 95\% CI for group comparison.

The incidence rates were higher for all studied diagnoses in the populations with T1D and T2D when compared with the population without DM, with the exception of OA of the CMC-1 joint among men with T1D (IRR 1.7, 95\% CI: 0.9 to $2.8, \mathrm{p}=0.055$ ). Trigger finger among women with T1D had the highest IR with 100.5 cases per 10000 person-years and an IRR of 8.1 (95\% CI: 7.1 to $9.1, \mathrm{p}<0.00001)$ compared with the population without DM. Finally, age-stratified and sex-stratified incidence rates for the respective diagnoses in 10-year age groups are presented in online supplemental figures S1-S5.

\section{DISCUSSION}

\section{Principal findings}

The analysis of the data from the SHR and the NDR from 2004 and 2019 showed markedly higher prevalences of all diagnoses studied, that is, CTS, TF, DD, UNE, and OA 


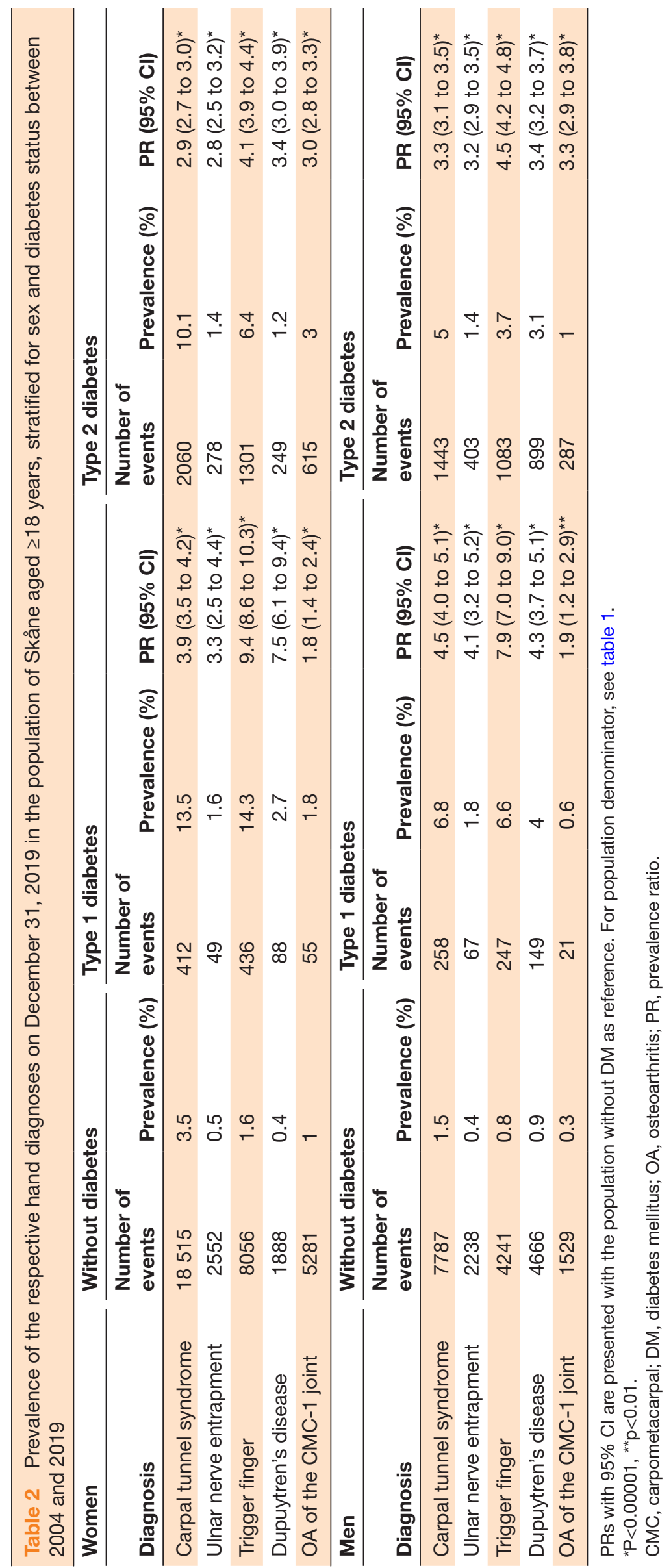




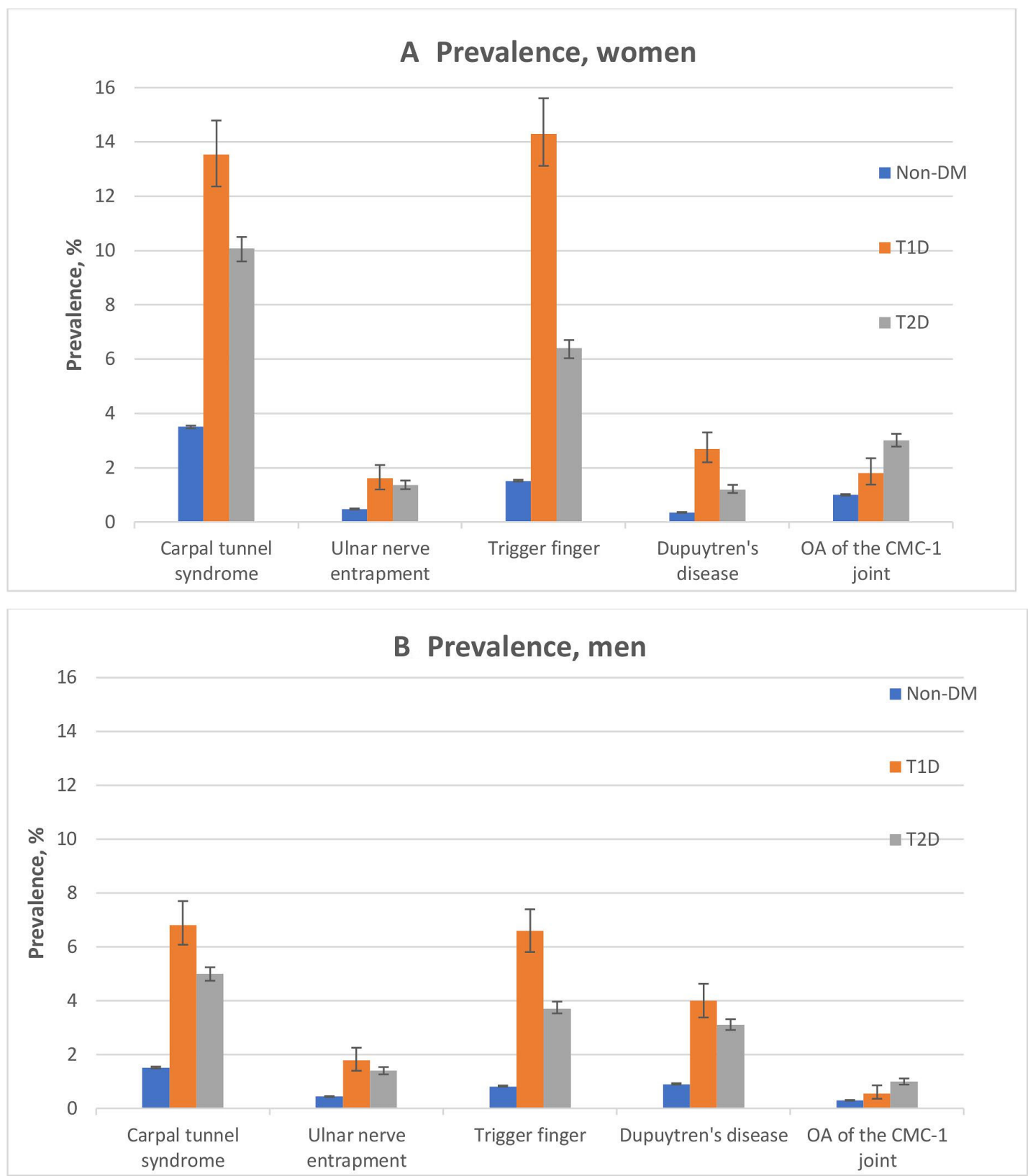

Figure 1 (A,B) Prevalence of hand diagnoses 2004-2019 in the population of Skåne aged $\geq 18$ years on December 31, 2019, stratified for sex and diabetes status, presented with 95\% Cls. CMC-1, first carpometacarpal; DM, diabetes mellitus; OA, osteoarthritis; T1D, type 1 diabetes; T2D, type 2 diabetes.

of the CMC-1 joint, among both men and women with T1D or T2D, compared with the population without DM.

Furthermore, both populations with T1D and T2D had more concomitant prevalent diagnoses when comparing with the population without DM. Finally, the 10-year incidence of all diagnoses was higher among the populations with T1D and T2D compared with the population without $\mathrm{DM}$, with the exception of OA of the CMC-1 joint among men with T1D. This study adds longitudinal, large-scale population-based data confirming that the entity 'the diabetic hand' should include both nerve compression syndromes, such as CTS and UNE, and disorders with impaired range of motion, like TF and DD, and possibly also OA of the CMC-1 joint. Care should be taken when examining patients with hand problems and concurrent $\mathrm{DM}$, bearing in mind the increased prevalences of all diagnoses in this study in the population with diabetes.

\section{Compression neuropathies}

The most common CN in the upper extremity is CTS, followed by UNE.

The present results show that the prevalences of both CTS and UNE are three to four times higher among both the populations with T1D and T2D, compared with the population without DM. These results are in line with several previous studies on DM and CTS. ${ }^{15} 16$ Although the epidemiological data on UNE are scarce, and the number of large cohort studies on UNE is limited, ${ }^{17}$ the 


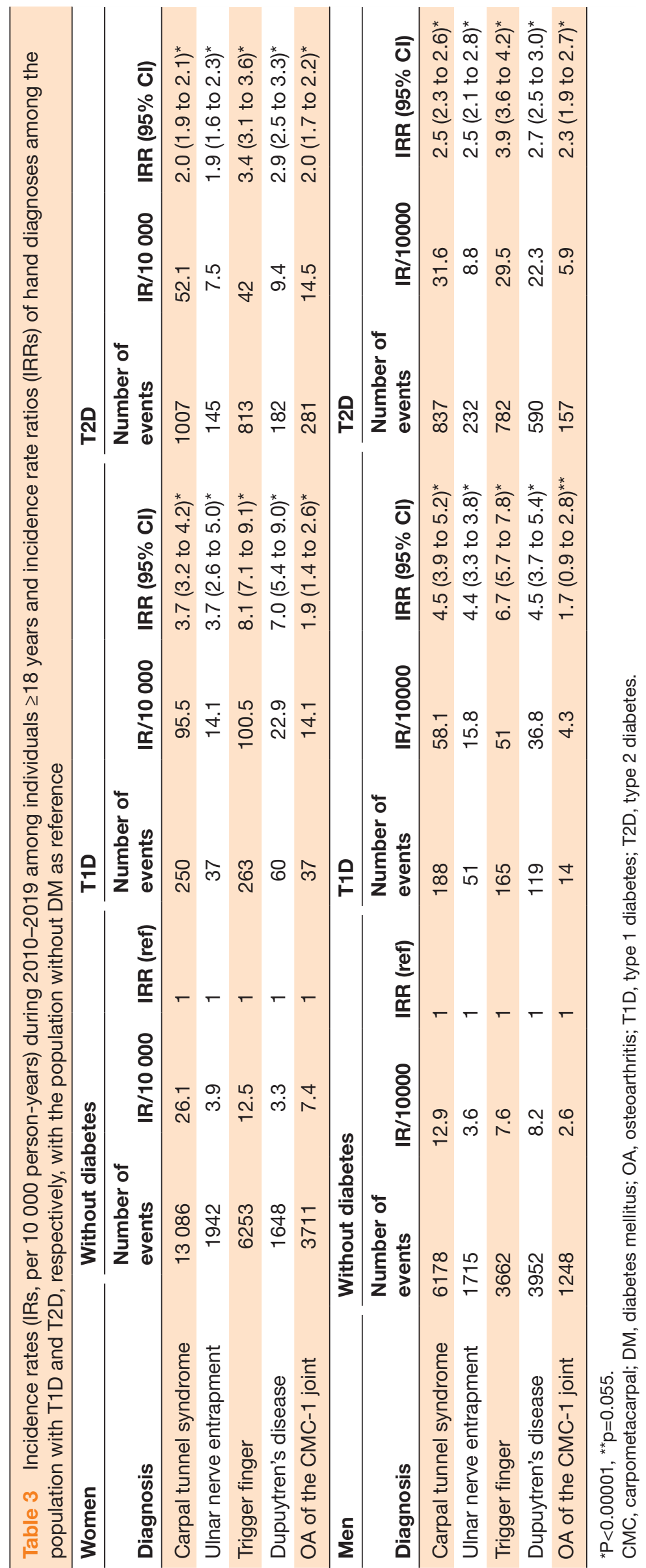


findings from this study corroborate both our previous work on identification of risk factors for $\mathrm{CN}^{4}$ and electrophysiological studies on UNE. ${ }^{318}$

Several structural and biochemical processes have been proposed as to why CNs are more common among the population with DM. These include deposition of glycated proteins, so called advanced glycation end products (AGEs), in the nerve and surrounding tissue possibly limiting nerve blood supply. ${ }^{19}{ }^{20}$ Furthermore, nerve swelling and edema due to deposition of intracellular hyperosmotic proteins, ${ }^{3}$ and alteration of the small microvessels surrounding the nerve, ${ }^{21}{ }^{22}$ might further decrease the blood supply to the nerve. Finally, biopsies from the posterior interosseous nerve among patients with DM and concurrent CTS have shown a reduction in nerve fiber density compared with controls, indicating a predisposition for nerve compressions among patients with DM. ${ }^{21}$

Taking this together, pathologic structural and biochemical alterations in the peripheral nerve and surrounding tissue due to DM and chronic hyperglycemia might lower the threshold for symptom development when the nerve is compressed, and thus increase the prevalences of CTS and possibly also UNE. ${ }^{4}$

\section{TF and DD}

Both TF and DD are considered to be fibroproliferative disorders with alterations in the flexor tendon pulley system $^{23}$ and palmar apeneurosis, ${ }^{24}$ respectively. The exact pathophysiology behind the diagnoses is still not fully understood, especially in the presence of $\mathrm{DM}{ }^{1}$ Nevertheless, several biochemical processes have been proposed in earlier studies as to why there is an increased risk of TF and DD among the population with DM. For DD, these include increased deposition of AGEs in the palmar fascia due to hyperglycemia and possible conversion of fibroblasts to myofibroblasts. ${ }^{125}{ }^{26}$ Regarding TF, a similar explanation has been proposed with chronic hyperglycemia leading to glycosylation and collagen deposition in the tendon sheet, ${ }^{1}$ possibly thickening the tendon sheet and particularly the A1 pulley. Alterations in collagen degradation due to chronic hyperglycemia have also been proposed. ${ }^{27}$ However, the number of biochemical studies on TF and DM is to the best of our knowledge very limited, making this issue an obvious target for further research.

The results from our study indicate that the prevalences of both TF and DD are marked higher among both the population with T1D and T2D. Over $14 \%$ of the women with T1D had a diagnosis of TF during 2004-2019; an incidence that was eight times higher compared with the population without DM. Similar results were found both among men with T1D and T2D. Our results corroborate several previous studies on the impact of DM on the risks of TF and $\mathrm{DD}^{2628-30}$; however, our study adds large-scale population-based data supporting that both TF and DD should be included in the concept of 'the diabetic hand'.

\section{OA of the CMC-1 joint}

OA of the CMC-1 joint is a common hand disorder, especially among elderly women. Symptoms include pain and weakness of the thumb, ultimately resulting in loss of function and mobility. ${ }^{31}$ OA has been thoroughly studied in the presence of DM yielding conflicting results, and a potential causal association between the diagnoses is yet to be established. ${ }^{52-35}$ Moreover, a BMI has been associated with OA, both of the hip and knee joint, but also with general hand OA and OA of the CMC-1 joint. ${ }^{36-38} \mathrm{At}$ the same time, a high BMI is a major risk factor for the development of $\mathrm{T} 2 \mathrm{D},{ }^{39}$ and also increasingly common among individuals with T1D. ${ }^{40}$

In our study, the prevalences of OA of the CMC-1 joint were higher among both men and women with T1D and T2D compared with the population without DM and, with the exception of men with T1D, the 10-year incidences were also higher among the populations with DM. Nevertheless, due to the observational, retrospective design of our study, we cannot draw any conclusions regarding causality of this finding. Thus, although the prevalence of OA of the CMC-1 joint was slightly higher among both individuals with T1D and T2D, the causal relationship between the diagnoses is still under debate and our results must be interpreted with this in mind.

In future studies aiming to investigate a potential causal link between DM and OA of the CMC-1 joint, BMI and other potential confounding factors that were not available in this study have to be taken into account and adjusted for. ${ }^{32}$

\section{Strengths and limitations}

The major strength of this study is the large, populationbased data on over 1.1 million inhabitants in the region of Skåne. The Swedish 10-digit personal identification number unique for every Swedish citizen, allowing crosslinkage of registries and large-scale epidemiological studies within this region, ${ }^{9}$ makes this study one of the largest epidemiological studies of diabetic hand complications. Another strength is the inclusion of data from primary care as well as from the NDR with nationwide coverage, enabling stratification for T1D and T2D. Studies only including data from specialized, hospitalbased care might underestimate the true prevalence of a diagnosis since not all cases are referred from a primary care physician. Our study included diagnoses from both primary care and hospital-based care, thus hopefully coming closer to the true prevalence of the diagnoses. However, our data do only reflect the prevalence and incidence of individuals diagnosed by a physician, and there are undeniably individuals who are only treated by an occupational therapist or do not receive healthcare at all for their hand symptoms. With this in mind, our results reflect clinically relevant data compared with studies investigating the prevalence in the general population, for example, survey studies. Furthermore, since all patients had been diagnosed by a physician, the case 
validity in our study ought to be high, compared with studies with self-reported data.

Nevertheless, due to the observational nature of the present data, it is important to mention that we are not able to draw any conclusions regarding casual relationships or pathophysiological mechanisms between DM and the diagnoses studied, even though their prevalences indeed were higher both among individuals with T1D and T2D. As previously discussed, there are confounding factors, for example, BMI, that might partly explain the present findings, or actually being a cause to development of, for example, neuropathy, rendering nerves more susceptible to compression.

The reported associations must therefore be interpreted together with previous studies, and meta-analyses would be helpful in order to be able to draw any conclusions regarding the impact of DM on the diagnoses studied.

Moreover, there might be additional diagnoses associated with the diabetic hand. For example, limited joint mobility, which in previous studies have been associated with $\mathrm{DM},{ }^{1}$ would have been interesting to be included in this study. Unfortunately, the ICD-10 code for LJT is not as well defined as for the other diagnoses studied, leading to difficulties registering the diagnosis in SHR. A case-control study on patients with T1D and T2D would probably be more suitable for studying LJT in Sweden.

Finally, the study only included data from 2004 to 2019, since the coverage rate of SHR drastically increased in $2004,{ }^{9}$ and consequently, patients diagnosed before 2004 were not included. Thus, the true prevalence might be higher than described in our data; however, the main results should not have been affected by this. Nevertheless, it is important to mention and should be kept in mind when interpreting the results from our study.

\section{CONCLUSION}

The study establishes CTS, UNE, TF, DD, and possibly also OA of the CMC-1 joint to be included in 'the diabetic hand' as all aforementioned diagnoses were more prevalent among both populations with T1D and T2D. Furthermore, both populations with T1D and T2D had more concomitant prevalent hand diagnoses when comparing with the population without DM. Future studies should try to elucidate the pathophysiology behind these increased prevalences among the population with DM in order to find potential therapeutic targets enabling prevention of diabetic complications in the hand.

\footnotetext{
Author affiliations

${ }^{1}$ Department of Translational Medicine-Hand Surgery, Lund University, Malmo, Sweden

${ }^{2}$ Hand Surgery, Skåne University Hospital, Malmo, Sweden

${ }^{3}$ Vascular Diseases, Skåne University Hospital, Malmo, Sweden

${ }^{4}$ National Diabetes Register, Centre of Registers in Region Västra Götaland, Gothenburg, Sweden

${ }^{5}$ Molecular and Clinical Medicine, Institute of Medicine, University of Gothenburg, Gothenburg, Sweden

${ }^{6}$ Department of Medicine, Sahlgrenska University Hospital, Gothenburg, Sweden
}

Contributors The study was designed and planned by MR, MZ, and LBD, and the initial draft of the manuscript was written by MR. All the authors discussed and contributed to intellectual content and to the data interpretation. All the stated authors reviewed and accepted the final version before publishing. MR and LBD are the guarantors of this study.

Funding This study was funded by Swedish Diabetes Foundation (DIA2020-492), the Regional Agreement on Medical Training and Clinical Research (ALF) between region Skåne and Lund University, Stig and Ragna Gorthons foundation (20191515), funds from Skåne University Hospital and local funds at Lund University.

Competing interests None declared.

Patient consent for publication Not required.

Ethics approval The study was approved by the Swedish national ethics committee (DNR: 2019-02042). The study was conducted in accordance with the Helsinki Declaration

Provenance and peer review Not commissioned; externally peer reviewed.

Data availability statement Data may be obtained from a third party and are not publicly available. Public access to the data used for this study is limited by the Swedish authorities, however, can be applied for by researchers after contacting the KVB committee in the region of Skåne (www.skane.se/en).

Supplemental material This content has been supplied by the author(s). It has not been vetted by BMJ Publishing Group Limited (BMJ) and may not have been peer-reviewed. Any opinions or recommendations discussed are solely those of the author(s) and are not endorsed by BMJ. BMJ disclaims all liability and responsibility arising from any reliance placed on the content. Where the content includes any translated material, BMJ does not warrant the accuracy and reliability of the translations (including but not limited to local regulations, clinical guidelines, terminology, drug names and drug dosages), and is not responsible for any error and/or omissions arising from translation and adaptation or otherwise.

Open access This is an open access article distributed in accordance with the Creative Commons Attribution Non Commercial (CC BY-NC 4.0) license, which permits others to distribute, remix, adapt, build upon this work non-commercially, and license their derivative works on different terms, provided the original work is properly cited, appropriate credit is given, any changes made indicated, and the use is non-commercial. See: http://creativecommons.org/licenses/by-nc/4.0/.

ORCID iDs

Mattias Rydberg http://orcid.org/0000-0002-8249-8660

Katarina Eeg-Olofsson http://orcid.org/0000-0002-3376-4707

\section{REFERENCES}

1 Papanas N, Maltezos E. The diabetic hand: a forgotten complication? J Diabetes Complications 2010;24:154-62.

2 Jennings AM, Milner PC, Ward JD. Hand abnormalities are associated with the complications of diabetes in type 2 diabetes. Diabetic Medicine 1989;6:43-7.

3 Rota E, Morelli N. Entrapment neuropathies in diabetes mellitus. World J Diabetes 2016;7:342-53.

4 Rydberg M, Zimmerman M, Gottsäter A, et al. Diabetes mellitus as a risk factor for compression neuropathy: a longitudinal cohort study from southern Sweden. BMJ Open Diab Res Care 2020;8:e001298.

5 Louati K, Vidal C, Berenbaum F, et al. Association between diabetes mellitus and osteoarthritis: systematic literature review and metaanalysis. RMD Open 2015;1:e000077.

6 World Health Organization. Global report on diabetes, 2016. Available: https://apps.who.int/iris/handle/10665/2048712019

7 Global Burden of Disease Collaborative Network. Global burden of disease study 2017 (GBD 2017) incidence, prevalence, and years lived with disability 1990-2017. Seattle, United States: Institute for Health Metrics and Evaluation (IHME), 2018.

8 Eliasson B, Gudbjörnsdottir S. Diabetes care - improvement through measurement. Diabetes Res Clin Pract 2014;106:S291-4.

9 Löfvendahl S, Schelin MEC, Jöud A. The value of the Skåne health-care register: prospectively collected individual-level data for population-based studies. Scand J Public Health 2020;48:56-63.

10 Rawshani A, Rawshani A, Franzén S, et al. Mortality and cardiovascular disease in type 1 and type 2 diabetes. New England Journal of Medicine 2017:376:1407-18.

11 Lind M, Bounias I, Olsson M, et al. Glycaemic control and incidence of heart failure in 20985 patients with type 1 diabetes: an observational study. The Lancet 2011;378:140-6. 
12 Sullivan KM. Two by two tables containing counts. Available: https:// www.openepi.com/PDFDocs/TwobyTwoDoc.pdf

13 Breslow NE, Day NE. Statistical methods in cancer research. volume II-The design and analysis of cohort studies. IARC Sci Publ 1987;82:1-406.

14 Dean AG SK, Soe MM. OpenEpi: open source epidemiologic statistics for public health, version, 2013. Available: www.OpenEpi. com [Accessed 16 Nov 2021].

15 Pourmemari $\mathrm{MH}$, Shiri R. Diabetes as a risk factor for carpal tunnel syndrome: a systematic review and meta-analysis. Diabetic Medicine 2016;33:10-16.

16 Comi G, Lozza L, Galardi G, et al. Presence of carpal tunnel syndrome in diabetics: effect of age, sex, diabetes duration and polyneuropathy. Acta Diabetol Lat 1985;22:259-62.

17 Rota E, Zavaroni D, Parietti L, et al. Ulnar entrapment neuropathy in patients with type 2 diabetes mellitus: an electrodiagnostic study. Diabetes Res Clin Pract 2014;104:73-8.

18 Acosta JA, Hoffman SN, Raynor EM, et al. Ulnar neuropathy in the forearm: a possible complication of diabetes mellitus. Muscle Nerve 2003;28:40-5.

19 Sessions J, Nickerson DS. Biologic basis of nerve decompression surgery for focal entrapments in diabetic peripheral neuropathy. $J$ Diabetes Sci Technol 2014;8:412-8.

20 Shimizu F, Sano Y, Haruki H, et al. Advanced glycation endproducts induce basement membrane hypertrophy in endoneurial microvessels and disrupt the blood-nerve barrier by stimulating the release of TGF- $\beta$ and vascular endothelial growth factor (VEGF) by pericytes. Diabetologia 2011;54:1517-26.

21 Thomsen NOB, Mojaddidi M, Malik RA, et al. Reduced myelinated nerve fibre and endoneurial capillary densities in the forearm of diabetic and non-diabetic patients with carpal tunnel syndrome. Acta Neuropathol 2009;118:785-91.

22 Feldman EL, Callaghan BC, Pop-Busui R, et al. Diabetic neuropathy. Nat Rev Dis Primers 2019;5:41.

23 Brozovich N, Agrawal D, Reddy G. A critical appraisal of adult trigger finger: pathophysiology, treatment, and future outlook. Plast Reconstr Surg Glob Open 2019;7:e2360-e60.

24 Dutta A, Jayasinghe G, Deore S, et al. Dupuytren's Contracture Current Concepts. J Clin Orthop Trauma 2020;11:590-6.

25 Takase F, Mifune Y, Inui A, et al. Association of advanced glycation end products in Dupuytren disease. J Orthop Surg Res 2018;13:143.

26 Broekstra DC, Groen H, Molenkamp S, et al. A systematic review and meta-analysis on the strength and consistency of the associations between Dupuytren disease and diabetes mellitus, liver disease, and epilepsy. Plast Reconstr Surg 2018;141:367e-79.
27 Blyth MJG, Ross DJ. Diabetes and trigger finger. Journal of Hand Surgery 1996;21:244-5.

28 Grandizio LC, Beck JD, Rutter MR, et al. The incidence of trigger digit after carpal tunnel release in diabetic and nondiabetic patients. $J$ Hand Surg Am 2014;39:280-5.

29 Chammas M, Bousquet P, Renard E, et al. Dupuytren's disease, carpal tunnel syndrome, trigger finger, and diabetes mellitus. J Hand Surg Am 1995;20:109-14.

30 Holte KB, Juel NG, Brox JI, et al. Hand, shoulder and back stiffness in long-term type 1 diabetes; cross-sectional association with skin collagen advanced glycation end-products. The Dialong study. $J$ Diabetes Complications 2017;31:1408-14.

31 Wolf JM, Turkiewicz A, Atroshi I, et al. Prevalence of doctordiagnosed thumb carpometacarpal joint osteoarthritis: an analysis of Swedish health care. Arthritis Care Res 2014;66:961-5.

32 Zhu Z, Sheng X, Zhang J, et al. Association between type 2 diabetes status and osteoarthritis in adults aged $\geq 50$ years. J Orthop Sci $2021 ; 37$.

33 Khor A, Ma C-A, Hong C, et al. Diabetes mellitus is not a risk factor for osteoarthritis. RMD Open 2020;6:e001030.

34 Williams MF, London DA, Husni EM, et al. Type 2 diabetes and osteoarthritis: a systematic review and meta-analysis. J Diabetes Complications 2016;30:944-50.

35 Funck-Brentano T, Nethander M, Movérare-Skrtic S, et al. Causa Factors for Knee, Hip, and Hand Osteoarthritis: A Mendelian Randomization Study in the UK Biobank. Arthritis Rheumatol 2019;71:1634-41.

36 Rydberg M, Dahlin LB, Gottsäter A, et al. High body mass index is associated with increased risk for osteoarthritis of the first carpometacarpal joint during more than 30 years of follow-up. $R M D$ Open 2020;6:e001368.

37 Jiang L, Xie X, Wang Y, et al. Body mass index and hand osteoarthritis susceptibility: an updated meta-analysis. Int J Rheum Dis 2016;19:1244-54.

38 Lohmander LS, Gerhardsson de Verdier M, Rollof J, et al. Incidence of severe knee and hip osteoarthritis in relation to different measures of body mass: a population-based prospective cohort study. Ann Rheum Dis 2009;68:490-6.

39 Schnurr TM, Jakupović H, Carrasquilla GD, et al. Obesity, unfavourable lifestyle and genetic risk of type 2 diabetes: a casecohort study. Diabetologia 2020;63:1324-32.

40 Corbin KD, Driscoll KA, Pratley RE, et al. Obesity in type 1 diabetes: pathophysiology, clinical impact, and mechanisms. Endocr Rev 2018;39:629-63. 